\title{
Application of Hydrazine Hydrate in the Synthesis of Octa(aminophenyl)silsesquioxane (OAPS) Poss
}

\author{
Jacob Kiptanui Koech, Qun Shao, Fredrick Nzioka Mutua, Yimin Wang \\ State Key Laboratory for Modification of Chemical Fibers and Polymer Materials, \\ College of Materials Science and Engineering, Donghua University, Shanghai, China \\ Email: Koech_jacob@yahoo.com
}

Received September 13, 2012; revised October 15, 2012; accepted October 24, 2012

\begin{abstract}
Octa(aminophenyl)silsesquioxane (OAPS) was prepared from octaphenyl silsesquioxane (OPS) in two steps, first nitration to obtain Octa(nitrophenyl)silsesquioxane (ONPS) then reduction by using the stable, inexpensive, and readily available hydrazine hydrate as the reducing agent in the presence of Iron(III)Chloride catalyst with a yield of around $87 \%$. Hydrazine is a two-electron reducing agent whereas nitro group is a four-electron reduction process. The activated carbon serves as an adsorbent and electrical conductor enabling the reaction to occur by acting as a mediator between a two-electron reagent and a four-electron process. Adsorption provides a reducing potential and a supply of electrons from many hydrazines making possible the initial four-electron process even though each individual hydrazine is a two-electron donor. The product was characterized by FTIR and ${ }^{1} \mathrm{H}$ NMR. The time period for preparation of ONPS from octaphenyl silsesquioxane was considerably shortened to avoid double nitration of the aromatic rings.
\end{abstract}

Keywords: Octa(aminophenyl)silsesquioxane; Octa(nitrophenyl)silsesquioxane; Hydrazine; Nanocomposite; Iron(III)Chloride; Reduction

\section{Introduction}

The growth of new materials with specially designed chemical, mechanical or physical properties is becoming increasingly important in many fields of practical application. In this respect, recent years have seen increased demand for organic-inorganic hybrid polymer materials in a wide range of applications [1]. These needs have been met with accelerated advances in polymer science and technology, taking benefit from nanophase technology geared towards enhancements to polymer materials. In order to accomplish the various practical requirements, a tailor-made material design is necessary, which includes direct control of both bulk and surface characteristics [2]. The nanostructures, degree of organization and properties that can be obtained from such materials certainly depend on the chemical nature of their components, but they also rely on the synergy between these components.

Polymer composites containing nanometer-size inorganic particles have received considerable attention from researchers in quest for materials with low density and much higher mechanical strength and stiffness compared to glass and carbon fiber filled counterparts [3]. A number of success stories give credence to the hypothesis that small quantities of, often less than $10 \mathrm{wt} \%$, inorganic nanoparticles can contribute to significant increases in heat distortion temperature, stiffness, and toughness of the polymer [4]. This is often attributed to the predominant interfacial interactions between the nanoparticles and polymer chains, which is facilitated by very large interfacial area per unit volume [5]. To illustrate, single wall carbon nanotubes (SWNT) have surface area $\sim 400$ $\mathrm{m}^{2} / \mathrm{g}$ [5], fumed silica $\sim 300 \mathrm{~m}^{2} / \mathrm{g}$, and Polyhedral oligomeric silsesquioxane (POSS) $\sim 406 \mathrm{~m}^{2} / \mathrm{g}$ [6]. In contrast to other nanofillers, such as layered silicates which are platy and carbon nanotubes which are cylindrical, the shape of the POSS molecules is isotropic. In addition, POSS molecules can be easily functionalized by chemically altering the R groups (Figure 1). This presents opportunities for copolymerization or grafting reactions with functional groups on polymer chains and for defining and controlling the nature of interactions between POSS molecules and the polymer chains. Some ramifications of such strong interactions are favorable rheological properties such as reduced shear viscosity, high glass transition, heat distortion temperatures, and improved mechanical properties of the nanocomposites.

Interest in Octa-aminophenyl polyhedral oligomeric silsesquioxane (OAPS) materials, shown in Figure 1, is based on the facts that the amino groups provide an excellent site for cross linking offering a unique opportu- 
nity for preparing truly molecularly dispersed nanocomposites $[7,8]$. POSS combine a hybrid inorganic-organic composition, $\mathrm{R}_{\mathrm{n}}(\mathrm{SiO} 1.5)_{\mathrm{n}}$, where $\mathrm{R}$ may be a range of organo-functional groups, while $\mathrm{n}$ is an even integer $\geq 4$, with nano-sized cage structures having dimensions comparable to those of most polymeric segments or coils [9]. POSS chemical reagents are nanostructured with sizes of $1-3 \mathrm{~nm}$ and can be thought of as the smallest particles of silica possible. However, unlike silica or silicones, each POSS molecule may contain nonreactive organic substituent that makes the POSS nanostructure compatible with monomers or polymers.

POSS molecules have been easily incorporated into common polymers via copolymerization, grafting or blending [10]. Varieties of POSS-containing copolymers have been prepared using condensation, ring-opening metathesis and radical, both conventional, and atomtransfer, copolymerization techniques. Many varieties of substituent may be fixed onto the $\mathrm{Si}$ atoms at the corners of the cages to optimize polymer-POSS interactions and simultaneously to aid in the easy dissolution of the POSS moieties in common solvents for synthesis purposes. These groups may be incorporated into almost any conventional polymer and in a variety of different chain architectures. Thus, inorganic nature and multiple reactive functionalities of POSS make these compounds ideal for their use in the construction of organic-inorganic hybrid nanomaterials [11].

Nowadays, most large-scale aromatic amines are being produced by catalytic hydrogenation of the corresponding nitroarenes. With a large variety of catalysts (e.g. Ni, $\mathrm{Cu}, \mathrm{Co}, \mathrm{Cr}, \mathrm{Fe}, \mathrm{Sn}, \mathrm{Ag}, \mathrm{Pt}, \mathrm{Pd}, \mathrm{Zn}, \mathrm{Ti}, \mathrm{Mo}$, metal oxides and sulphides) $[12,13]$ and under a wide range of reaction conditions in most cases the corresponding amine is obtained quantitatively without the production of waste products $[14,15]$. Because of the exothermic nature of the reaction and the need for a closed high-pressure system, numerous safety precautions have to be taken.

Despite such optimistic outlook and well executed prior work, the cost of OAPS is exorbitantly high calling for ways of producing the same cheaply. One way of achieving this is by replacing the conventional method of synthesizing OAPS by use of $\mathrm{Pd} / \mathrm{C}$ with hydrazine hydrate which is cheaply available.

This paper explores a novel synthesis of OAPS in two steps from octaphenyl POSS as shown in Figure 2. First,
OPS is subjected to nitration to obtain ONPS by use of fuming nitric acid. ONPS is then subjected to mild reduction using hydrazine hydrate to obtain OAPS.

\section{Experimental Details}

\subsection{Materials}

OPS was purchased from Laoning AM union composite materials Co. Ltd. Tetrahydrofuran and ethyl acetate were obtained through Donghua university chemical store, dried over molecular sieve prior to use. Other chemicals and reagents were also sourced through Donghua university chemical store and used as received.

\subsection{Synthesis of Octanitrophenyl POSS}

Octa(nitrophenyl)-POSS (ONP-POSS) was synthesized by adopting a procedure previously reported by Laine and coworkers with a number of modifications [16]. A $20 \mathrm{~g}$ of OP-POSS $(19.4 \mathrm{mmol})$ was added in small portion to $135 \mathrm{~mL}$ fuming nitric acid while stirring at $0^{\circ} \mathrm{C}$. The resulting mixture was kept in an ice cold condition for an hour then at room temperature with constant agitation for $7 \mathrm{~h}$. The solution was then poured onto $150 \mathrm{~g}$ ice and a faintly yellow precipitate collected, washed with water (until $\mathrm{pH}$ 6.0) then with ethanol $(200 \mathrm{~mL} \times 2)$. The powder of ONP-POSS was obtained by filtration and dried under vacuum at $50^{\circ} \mathrm{C}$ for $12 \mathrm{hrs}$. (yield 91\%).

\subsection{Synthesis of Octaaminophenyl POSS}

Five grams of ONPS (3.86 mmol), $50 \mathrm{mg} \mathrm{FeCl}$, and $4 \mathrm{~g}$ active charcoal powder were charged into a three necked $250 \mathrm{~mL}$ round-bottomed flask equipped with a magnetic

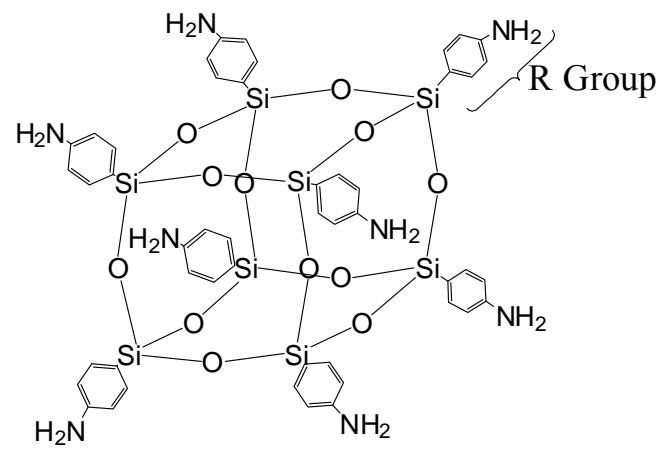

Figure 1. Octa-aminophenyl POSS molecule.

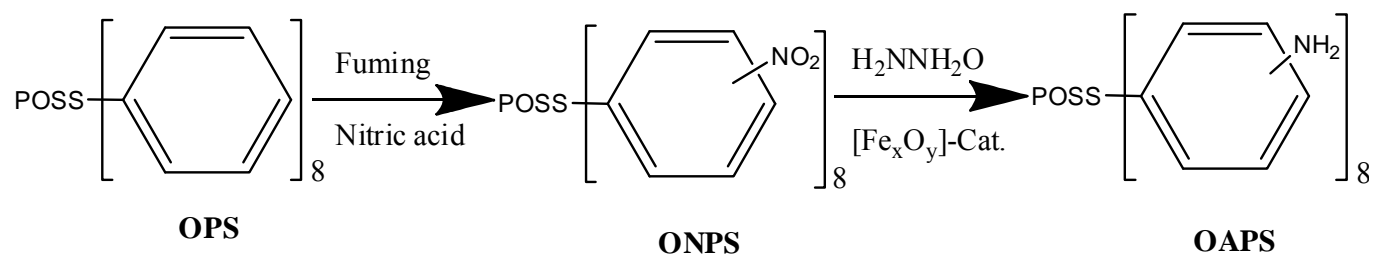

Figure 2. Synthesis of OAPS. 
stirrer and a condenser. THF $(40 \mathrm{~mL})$ was then added to the flask. The solution was stirred and heated to $60^{\circ} \mathrm{C}$ under $\mathrm{N}_{2}$.

Hydrazine hydrate $(16 \mathrm{~mL})$ was added drop-wise into the mixture. The reaction was continued for $4 \mathrm{~h}$, and then the solution was cooled and filtered. The filtrate was combined with $25 \mathrm{~mL}$ of ethyl acetate and washed with water thrice. The organic layer was dried over $\mathrm{Na}_{2} \mathrm{SO}_{4}$ and poured into $1 \mathrm{~L}$ petroleum ether. The white precipitate was collected by filtration. The product was redissolved in the mixture of $15 \mathrm{~mL}$ THF and $25 \mathrm{~mL}$ ethyl acetate, and reprecipitated into $500 \mathrm{~mL}$ petroleum ether. The obtained powder was dried in vacuum at $50^{\circ} \mathrm{C}$. The yield of the off-white OAPS was $87 \%$.

\subsection{Measurement and Characterization}

Fourier transform infrared (FTIR) spectra of the samples were recorded on a Nicolet 5700 FTIR (Thermo Fisher) in which $\mathrm{KBr}$ pellets were used for the powder samples of the synthesized monomer. ${ }^{1} \mathrm{H}$ NMR spectra were recorded on a Bruker-400 spectrometer operating at 296.2 $\mathrm{K}$ with tetramethylsilane (TMS) as an internal reference. The spectra were referenced to the residual proton impurities in the $\mathrm{CDCl}_{3}$ at $\delta 7.27 \mathrm{ppm}$.

\section{Discussion and Conclusions}

The OAPS was prepared as depicted in Figure 2, and its structure was characterized. In the FTIR spectrum of ONPS (Figure 3(b)), the two strong peaks at 1350 and $1531 \mathrm{~cm}^{-1}$ are assigned to symmetric and asymmetric $\mathrm{vN}=\mathrm{O}$. These two peaks disappeared after reduction as shown in the FTIR spectrum of OAPS (Figure 3(c)), denoting that the reduction reaction was successful. New broad peaks appear at $3381 \mathrm{~cm}^{-1}$ with a shoulder at 3223 $\mathrm{cm}^{-1}$, which are assigned to vNH $[17,18]$.

In the 1H NMR spectrum of OAPS (Figure 4(a)), no peak at $8-9$ ppm corresponding to the protons in aromatic groups of ONPS was observed, while the aromatic hydrogen of OAPS were detected at higher magnetic field $(7.8-6.0 \mathrm{ppm})$ due to the electron donor properties of amino groups on phenyl rings. This result indicated that the reduction reaction of nitro groups in ONPS by hydrazine hydrate was complete. The integration ratio of the peaks originating from protons in aromatic groups and amino groups of OAPS results indicated that one hydrogen atom per phenyl in OPS was substituted by nitro group forming ONPS, which was subsequently converted into amine group forming OAPS by reduction reaction. The $\mathrm{Si}-\mathrm{O}-\mathrm{Si}$ bonds in silsesquioxane cages of OAPS could be characterized by the stretching bands at $1119 \mathrm{~cm}^{-1}$ in FTIR spectrum (Figure 3) [17,19].

The reduction of aromatic nitro compounds with hydrazine or hydrazine hydrate, illustrated in Figure 5, represents a special variation of the catalytic reduction, where hydrazine is the source of the hydrogen. The hydrogen can be generated by a variety of H-transfer catalysis [13]. Especially with the use of noble metal catalysts, such as $\mathrm{Pd}, \mathrm{Pt}$ or $\mathrm{Ru}$, but also with the application of $\mathrm{Ni}, \mathrm{Cu}$ or $\mathrm{Fe}$, the catalytic hydrazine reduction gives high yields comparable to or better than the catalytic hydrogenation.

The following chemical reaction illustrates the mechanism by which hydrazine hydrate is broken down by catalytic iron to release hydrogen ions in the presence activated carbon.

In the past, the relatively high costs for hydrazine hydrate and for the noble metal catalysts prevented this reduction method from being applied at an industrial scale. However, there are two main reasons which are currently enhancing the preference of this catalytic $\mathrm{H}$ transfer reduction: 1) It has been observed repeatedly that several cheap iron(III) compounds exhibit an appreciable activity with regard to catalytic H-transfer [20]; 2) In cases where the catalytic hydrogenation is not the method of choice, this method offers a safe as well as an ecologically and economically beneficial alternative, above all for small product volumes in chemical manufacture, where the reaction can be carried out in multiuse batches under normal pressure.

\section{Conclusions}

Hydrazine hydrate catalytic reduction under iron(III) chloride/activated carbon provides an efficient and relatively cheap method for the production of OAPS. Iron(III) chloride is directly involved in the reaction as active centre where as the activated carbon acts as the anchorage which helps in adsorption of hydrazine and transfer of electrons to nitro groups ensuring a smooth reaction progress, it basically acts as a stabilizer. Hydrazine is a convenient reductant because the by-products are typically

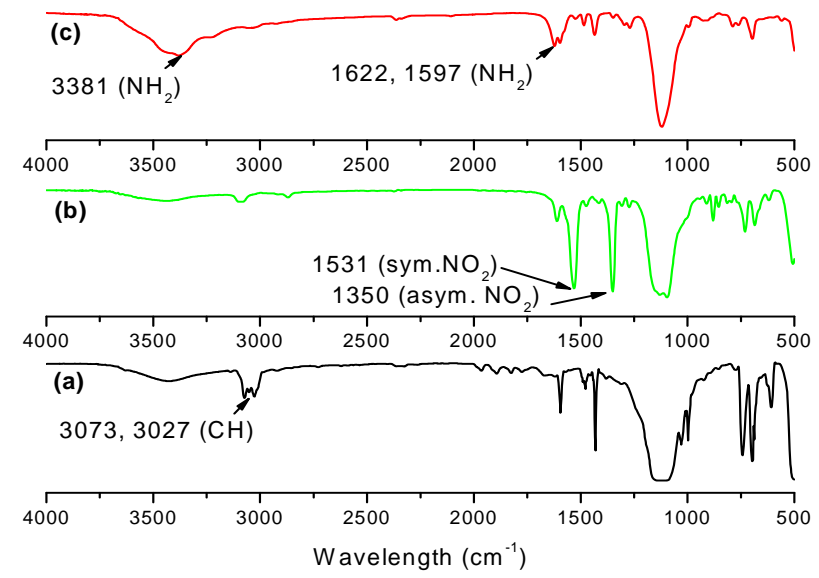

Figure 3. FTIR spectrum of (a) OPS, (b) ONPS and (c) OAPS. 

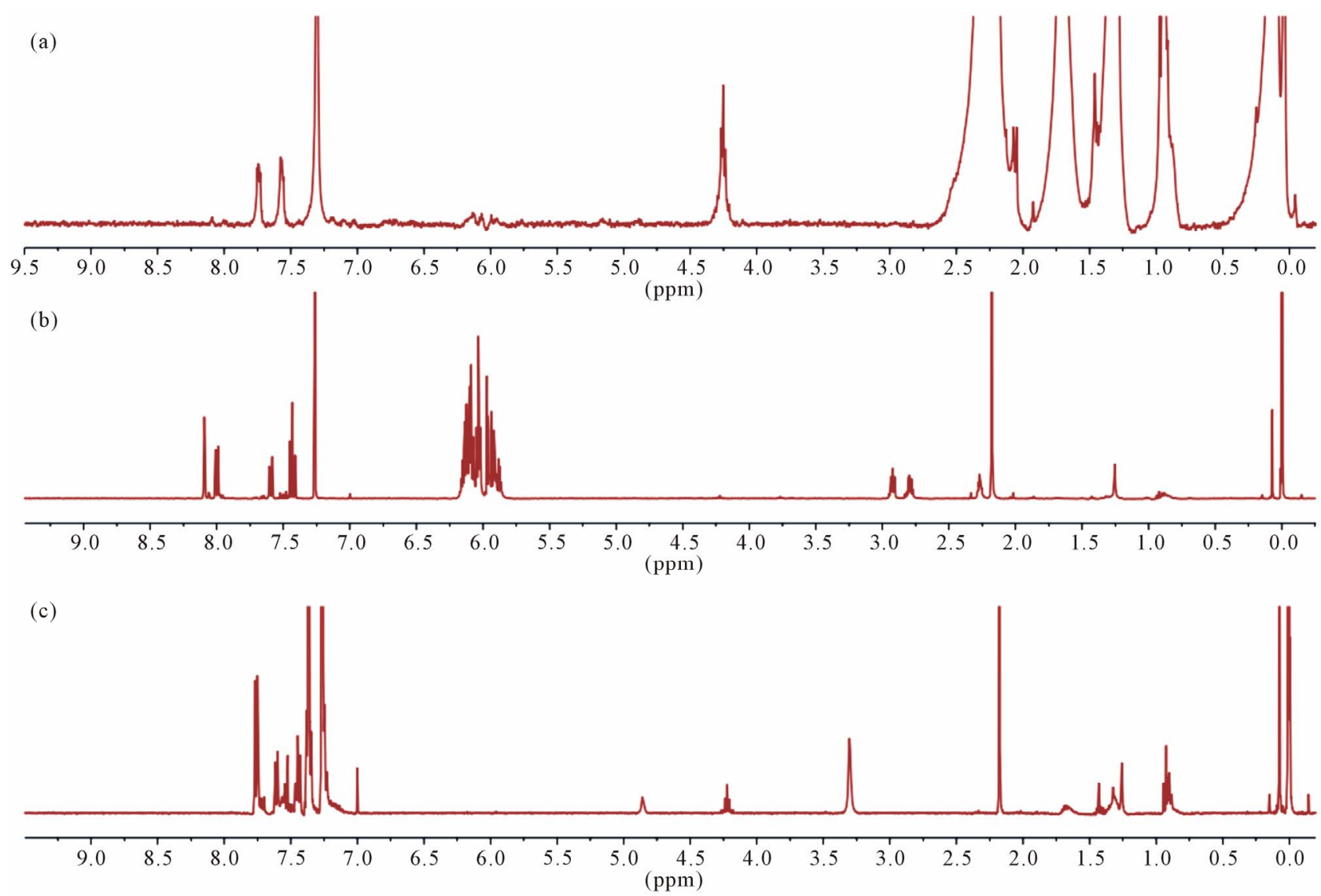

Figure 4. HNMR spectrum of (a) OAPS, (b) ONPS and (c) OPS.

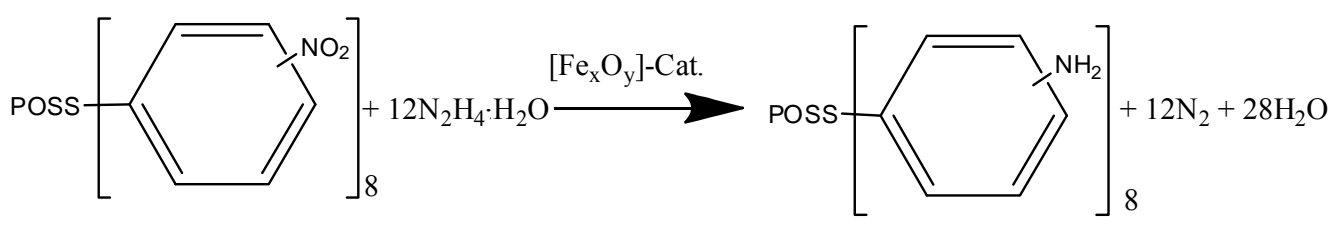

Figure 5. Catalytic reduction of ONPS to obtain OAPS.

nitrogen gas and water (Figure 5).

The direct reaction of hydrazine and nitroaromatics does not occur because of a mismatch in the electronic requirements, a two-electron reductant and a four electron oxidant. Activated carbon facilitates the reaction by providing a pool of electrons from adsorbed hydrazine. It serves as an adsorbent and electrical conductor. The rate determining step is oxidation of hydrazine at the carbon surface.

\section{Acknowledgements}

The authors gratefully acknowledge Zhang Yao for his tireless help in the execution of the experiments.

\section{REFERENCES}

[1] E. Markovic, K. Constantopolous and J. G. Matisons, "Po- lyhedral Oligomeric Silsesquioxanes: From Early and Strategic Development through to Materials Application," Applications of Polyhedral Oligomeric Silsesquioxanes, 2011, pp. 1-46.

[2] S. Lucke and K. Stoppek-Langner, "Polyhedral Oligosilsesquioxanes (POSS) - Building Blocks for the Development of Nano-Structured Materials," Applied Surface Science, Vol. 144-145, 1999, pp. 713-715. doi:10.1016/S0169-4332(98)00912-X

[3] S.-W. Kuo and F.-C. Chang, "POSS Related Polymer Nanocomposites," Progress in Polymer Science, Vol. 36, No. 12, 2011, pp. 1649-1696. doi:10.1016/j.progpolymsci.2011.05.002

[4] K. Pielichowski, et al., "Polyhedral Oligomeric Silsesquioxanes (POSS)-Containing Nanohybrid Polymers," Supramolecular Polymers Polymeric Betains Oligomers, Vol. 201, 2006, pp. 225-296. doi:10.1007/12_077

[5] I. A. A. C. Esteves, et al., "Determination of the Surface Area and Porosity of Carbon Nanotube Bundles from a 
Langmuirian Analysis of Sub- and Supercritical Adsorption Data," Carbon, Vol. 47, No. 4, 2009, pp. 948-956. doi:10.1016/i.carbon.2008.11.044

[6] R. A. Mantz, et al., "Thermolysis of Polyhedral Oligomeric Silsesquioxane (POSS) Macromers and POSS-Siloxane Copolymers," Chemistry of Materials, Vol. 8, No. 6, 1996, pp. 1250-1259. doi:10.1021/cm950536x

[7] G. Z. Li, et al., "Viscoelastic and Mechanical Properties of Epoxy/Multifunctional Polyhedral Oligomeric Silsesquioxane Nanocomposites and Epoxy/Ladderlike Polyphenylsilsesquioxane Blends," Macromolecules, Vol. 34, No. 25, 2001, pp. 8686-8693. doi:10.1021/ma011117q

[8] C. Ramirez, et al., "Selection of a Precursor of a Monofunctional Polyhedral Oligomeric Silsesquioxane Reacted with Aromatic Diamines," Journal of Applied Polymer Science, Vol. 92, No. 3, 2004, pp. 1576-1583. doi:10.1002/app.20101

[9] W. A. Reinerth, et al., "POSS (TM)/Polymer Blends for Gas Separation," Abstracts of Papers of the American Chemical Society, Vol. 222, 2001, p. U429.

[10] E. G. Shockey, et al., "Functionalized Polyhedral Oligosilsesquioxane (POSS) Macromers: New Graftable POSS Hydride, POSS Alpha-Olefin, POSS Epoxy, and POSS Chlorosilane Macromers and POSS-Siloxane Triblocks," Applied Organometallic Chemistry, Vol. 13, No. 4, 1999, pp. 311-327. doi:10.1002/(SICI)1099-0739(199904)13:4<311::AID-A OC847>3.0.CO;2-1

[11] D. Neumann, et al., "Synthesis and Characterization of an Isocyanate Functionalized Polyhedral Oligosilsesquioxane and the Subsequent Formation of an Organic-Inorganic Hybrid Polyurethane," Journal of the American Chemical Society, Vol. 124, No. 47, 2002, pp. 13998-13999. doi:10.1021/ja0275921

[12] M. Lauwiner, R. Roth and P. Rys, "Reduction of Aromatic Nitro Compounds with Hydrazine Hydrate in the Presence of an Iron Oxide/Hydroxide Catalyst. III. The Selective Reduction of Nitro Groups in Aromatic Azo Compounds," Applied Catalysis A: General, Vol. 177, No. 1, 1999, pp. 9-14. doi:10.1016/S0926-860X(98)00247-6
[13] M. Lauwiner, P. Rys and J. Wissmann, "Reduction of Aromatic Nitro Compounds with Hydrazine Hydrate in the Presence of an Iron Oxide Hydroxide Catalyst. I. The Reduction of Monosubstituted Nitrobenzenes with Hydrazine Hydrate in the Presence of ferrihydrite," Applied Catalysis A: General, Vol. 172, No. 1, 1998, pp. 141-148. doi:10.1016/S0926-860X(98)00110-0

[14] M. Benz and R. Prins, "Kinetics of the Reduction of Aromatic Nitro Compounds with Hydrazine Hydrate in the Presence of an Iron Oxide Hydroxide Catalyst," Applied Catalysis A: General, Vol. 183, No. 2, 1999, pp. 325-333. doi:10.1016/S0926-860X(99)00069-1

[15] M. Benz, A. M. van der Kraan and R. Prins, "Reduction of Aromatic Nitrocompounds with Hydrazine Hydrate in the Presence of an Iron Oxide Hydroxide Catalyst: II. Activity, X-Ray Diffraction and Mössbauer Study of the Iron Oxide Hydroxide Catalyst," Applied Catalysis A: General, Vol. 172, No. 1, 1998, pp. 149-157. doi:10.1016/S0926-860X(98)00111-2

[16] S. G. Kim, et al., "Synthesis of Amino-Containing Oligophenylsilsesquioxanes," Polymer, Vol. 46, No. 12, 2005, pp. 4514-4524. doi:10.1016/j.polymer.2005.02.036

[17] Y. Ni and S. Zheng, "A Novel Photocrosslinkable Polyhedral Oligomeric Silsesquioxane and Its Nanocomposites with Poly (Vinyl Cinnamate)," Chemistry of Materials, Vol. 16, No. 24, 2004, pp. 5141-5148. doi: $10.1021 / \mathrm{cm} 049463 \mathrm{k}$

[18] R. Tamaki, et al., "Octa(Aminophenyl) Silsesquioxane as a Nanoconstruction Site," Journal of the American Chemical Society, Vol. 123, No. 49, 2001, pp. 12416-12417. doi: $10.1021 / \mathrm{ja} 011781 \mathrm{~m}$

[19] R. Tamaki, J. Choi and R. M. Laine, "A Polyimide Nanocomposite from Octa(Aminophenyl) Silsesquioxane," Chemistry of Materials, Vol. 15, No. 3, 2003, pp. 793-797. doi: $10.1021 / \mathrm{cm} 020797 \mathrm{o}$

[20] U. Schwertmann and R. M. Cornell, "Iron Oxides in the Laboratory: Preparation and Characterization," 2nd Edition, Wiley-VCH, Weinheim, 2000. 